\title{
Blood group linked to risk of cancer in patients with Barrett esophagus
}

"Patients with Barrett esophagus who are blood group type $\mathrm{O}$ and Rhesus $\mathrm{D}$ $(\mathrm{RhD})$ negative are at particular risk of disease progression to esophageal cancer, and perhaps should be under increased surveillance," says Christine Caygill from the Royal Free Hospital, UK, corresponding author of a recent study on the topic.

There is a long history of studying the association between blood groups and disease, the rationale being that an associated blood group may be a surrogate marker for an underlying genetic mutation.

Researchers from the UK and the US examined the blood group distribution pattern across the full spectrum of patients with GERD-ranging from those with nonerosive esophagitis to those with esophageal cancer-and compared it with that of healthy blood donors. The goal was to identify any association between blood group and the incidence of disease.
Individuals who were $\mathrm{RhD}$ positive had approximately the same pattern of blood group distribution as the healthy blood donating population, whereas those who were $\mathrm{RhD}$ negative (the minority) did not. The most striking finding was a significant correlation between progression from Barrett esophagus to esophageal cancer in patients with GERD who were blood type $\mathrm{O}$ and $\mathrm{RhD}$ negative.

The team believe that gas transport, specifically nitric oxide transport, which is the principle function of the $\mathrm{RhD}$ factor, may offer insight into a possible mechanism of disease for this association. The group hope to explore this theory in their future work.

\section{Rachel Thompson}

Original article Caygill, C. P. et al. Barrett's, blood groups and progression to oesophageal cancer: is nitric oxide the link? Eur. J. Gastroenterol. Hepatol. doi:10.1097/ MEG.0b013e3283489dcf 